\title{
Las teorías criminológicas \\ y la llegada del sistema \\ Bertillon a la ciudad de \\ México, ¿un caso de éxito?
}

\section{Karina Villegas Terán ${ }^{1}$ karyjed@gmail.com}

Criminological theories and the arrival

of the Bertillon system to Mexico City. A

\section{Resumen}

A finales del siglo XIX, la llegada de teorías criminológicas a México impulsó la introducción de nuevas técnicas para la identificación de los criminales. Una de ellas fue el sistema Bertillon, que se esperaba sirviera para definir, mediante preceptos científicos, quién y cómo era físicamente el criminal. Dicho sistema cambió la forma en que los criminales fueron percibidos por los estudiosos de la criminología, lo cual, como se verá, suscitó diferentes polémicas en torno a cómo debía ser tratado el delincuente, así como la posibilidad que tenía de regenerarse.

Palabras clave: sistema Bertillon, cárcel de Belem, antropometría, presos, criminalidad.

\section{Abstract}

In the last years of the nineteenth century the arrival of criminological theories in Mexico impulse the introduction of new techniques for the criminal identification. Once of this was the Bertillon system, that the people hoped, serve to define, was the criminal. This system changed the forms that criminals were perceived by students of criminology, which, as will be seen, raised various controversies about the treat of criminals, and the posibilities that they have for regenerate.

Key words: Bertillon system, Belem prison, anthropometry, prisoners, crime, nineteenth century. Universidad Nacional Autónoma de México, México. Facultad de Filosofía y Letras. Circuito interior s/n Col. Universidad Nacional Autónoma de México, Coyoacán, C.P. 04510, México, D. F. 


\section{Introducción ${ }^{2}$}

La madrugada del 7 de enero de 1892, en la Cárcel Nacional, el preso Jesús Bruno Martínez fue conducido al patio del jardín, un "fatídico paredón, picoteado con las balas que acabaron con la vida de quién sabe cuántos hombres". ${ }^{3}$ Ahí se reunieron alrededor de veinte individuos, entre periodistas y empleados de la cárcel, que deseaban presenciar el fusilamiento de Martínez. El condenado fue sentenciado a la pena de muerte tras asaltar una joyería del centro y matar al dueño -el señor Hernández-, hecho que se conoció popularmente como "el crimen de la Profesa".

Bruno Martínez iba custodiado al paredón cuando, en un intento de huir de su destino, sacó un chuchillo e hirió al $2^{\circ}$ jefe de la policía secreta, Miguel Cabrera, quien murió rápidamente. Por unos momentos el caos reinó. El próximo a ser fusilado intentó huir mientras lanzaba cuchilladas por doquier. Su esfuerzo fue en vano, pues se le acorraló y ajustició en el mismo instante. Segundos después, el doctor Ignacio Fernández Ortigosa recogía la cabeza y el cuerpo de Martínez para realizar la autopsia y los exámenes correspondientes.

Los exámenes, se creía, no sólo determinarían la causa de su muerte, sino que ayudarían a esclarecer los motivos por los cuales Martínez había llegado a ser un criminal. Durante el porfiriato, ese modo de proceder con el cuerpo no era algo aislado, sino que resultaba ser algo común para médicos y abogados que se ocupaban de establecer quién era el criminal y las razones por las cuales había llegado a transgredir el orden. En las siguientes líneas se analizará la forma en la que los criminalistas mexicanos trataron de encontrar al tipo de criminal mexicano, durante las últimas décadas del siglo XIX, mediante diversas teorías que pueden ser divididas en dos grupos: la escuela clásica o liberal y la positivista.

\section{Las escuelas criminalistas}

La escuela clásica postulaba que el individuo tenía libre albedrío en el momento de actuar y por ende una responsabilidad moral por sus acciones u omisiones, que debían ser juzgadas de igual forma entre todos los

\footnotetext{
Agradezco a la doctora Georgette José por todo el apoyo que recibí de su parte para escribir este artículo, así como a Ana María T. Alejos por su preocupación y cariño constantes. El artículo esta dedicado al licenciado Francisco Méndez, por sus críticas, su lectura y su tiempo. Javier Piña Palacios, "Estado de la Cárcel Nacional conocida como Cárcel de Belem en el año de 1882", Criminalia, México, agosto de 1959, núm. 8, p. 424.
} 
individuos. ${ }^{4}$ La escuela planteaba que el delito era un acto incorrecto que podía cometer cualquiera, mientras que todo delincuente podría regenerarse con la ayuda de la instrucción. ${ }^{5}$

Por su parte, los positivistas argumentaban que el criminal era un ser predeterminado a delinquir desde su nacimiento y que la forma de castigarlo debía atender a "una serie de medidas adecuadas a la resolución de los hechos sociales", ${ }^{6}$ a las que se llegaría mediante el análisis de un conjunto de causas y efectos que se repetían en la sociedad invariablemente. Se postulaba que el conocimiento físico y científico del delincuente ayudaría a establecer las causas que lo conducían al delito, porque aseguraban que todo pensamiento positivo debía justificarse mediante alguna teoría que pudiera comprobarse, pues "el núcleo de la filosofía positivista es la búsqueda de un número siempre decreciente de leyes o 'hechos generales' de los cuales todos los fenómenos observables son casos concretos". ${ }^{7}$

Dicha escuela tuvo sus orígenes en la frenología, la cual surgió en Europa en el siglo XVIII. La frenología señalaba que el cerebro poseía veintisiete facultades diferentes, que daban forma al contorno craneal. Mientras más grande era determinada parte del cerebro, ello significaba que el sujeto poseía desarrollada la habilidad o sentimiento que en dicho lugar se encontraba. De igual forma, a mayor depresión en los ángulos craneales, el individuo carecía de las habilidades correspondientes. ${ }^{8}$

La frenología tuvo éxito entre los médicos mexicanos de mediados del siglo XIX, mientras que su sucesora triunfó entre los positivistas mexicanos, pues lo que calificaban como determinismo se ajustaba a la perfección con la idea de que existían factores físicos que "construían" al criminal. Entre los principales defensores de esta escuela se encontraban Justo Sierra, Rafael de Zayas Enríquez, Carlos Díaz, Carlos Roumagnac, Francisco Martínez Baca y Manuel Vergara, ${ }^{9}$ quienes se dieron a la tarea de poner en práctica los postulados de esta escuela en México. En 1892 Martínez Baca y Manuel Vergara realizaron un estudio en la cárcel de Puebla a partir de la instalación de uno de los primeros gabinetes antropométricos; Carlos Roumagnac hizo lo propio en la cárcel de Belem entre 1903 y 1904.

La escuela antropológica criminal nació en Italia con el médico César Lombroso. Hacia 1860, Lombroso formaba parte de la milicia del Piamonte

\footnotetext{
4

Padilla, De Belem, p. 97.

Speckman, Crimen, pp. 86 y 87.

Speckman, Crimen, p. 80.

Hale, La transformación, p. 58.

Castañeda, "La frenología", pp. 242- 246.

$9 \quad$ Speckman, Crimen, pp. 95-97.
} 
que se encargaba de perseguir bandidos, que siempre le atrajeron como objeto de estudio. En 1870 volvió al campo de la medicina, que había abandonado, en el Hospital de Santa Eugenia de Pavía, donde se especializó en el departamento para locos. Fue ahí donde, al observar el cráneo de un bandido famoso de apellido Villela, se propuso estudiar el origen de la delincuencia en las deformaciones craneanas. Escribió Lombroso: "el problema de la naturaleza del delito se me apareció súbitamente iluminado $[\ldots]$ entre alienados y criminales no había diferencia de calidad, sino de grado. Unos y otros eran atávicos" ${ }^{10}$ Con base en estas ideas realizó un estudio en 400 criminales. Los resultados se publicaron en 1876 con el título de L'Uomo delinquente y dio lugar a que en 1886 se celebrara el primer congreso de antropología criminal, en el que Lombroso propuso reformas al código penal italiano a partir de sus nuevos descubrimientos. Sin embargo, la idea no fue aceptada.

L'Uomo delinquente fue traducido al español con el título de Los criminales. Lombroso explicaba con argumentos "científicos" -por su método riguroso y por la comprobación del mismo con base en el estudio de los reos italianos-, que los rasgos fisiológicos de cada criminal correspondían y determinaban el tipo de crimen que podía cometer, pues desde su nacimiento el delincuente estaba destinado a serlo sin posibilidad de cambiar. Ejemplo de estas ideas fue el estudio que hizo sobre las características de la nariz de los delincuentes, donde concluía que la de los "normales" casi nunca era desviada. Este tipo de afirmaciones las buscó comprobar utilizando estadísticas:

Entre los criminales se ha podido determinar suficientemente la nariz del ladrón y la del estuprador. El ladrón presenta, en su mayoría, la nariz rectilínea (40.4 por 100); en bastantes casos cóncava (23.32 por 100 ); con base frecuentemente levantada (32.13 por 100); en muchas ocasiones, corta (30.92 por 100); larga (53.28 por 100); aplastada (31.33 por 100); y algunas veces desviada (37.6 por 100). Los estupradores tienen, casi siempre, la nariz rectilínea (54.5 por 100 ), aplastada (50 por 100) y desviada (50 por 100), pero de medianas dimensiones [...]. Adviértase por consiguiente, que si el perfil rectilíneo y la dirección desviada distinguen la nariz del criminal de la del normal, la longitud, latitud y protuberancia caracterizan suficientemente entre sí a los diferentes tipos de criminales. ${ }^{11}$

\footnotetext{
10 Luis Garrido, "El cincuentenario de Lombroso", Criminalia, México, octubre de 1959, Año XXv, núm. 10, p. 580.

11 Lombroso, Los criminales, p. 23
} 
Además, Lombroso denunció que en las cárceles no se realizaba un estudio adecuado de los criminales, ya que a diferencia de lo que ocurría con ellos, en el caso de los enfermos primero se estudiaba a éstos y no a la enfermedad. Lo mismo debía aplicarse al caso de los criminales; es decir, el análisis del criminal debía preceder al estudio del crimen. El estudio sería como un análisis clínico. Se empezaría por preguntar antecedentes, así como la forma en que había vivido hasta ese momento, lo que se acompañaría de un examen antropométrico y otro psicológico. Con base en esto se podría saber qué castigo merecía el criminal y cuál era el mejor para él, pues consideraba un error destinar al transgresor a celdas indeterminadas, ya que muchas veces esto le ocasionaba un mayor daño. ${ }^{12}$

En México, Carlos Roumagnac fue uno de los seguidores de las ideas de Lombroso y decidió hacer su propio estudio, que publicó con el título Los criminales en México. En él aplicó el análisis de la escuela italiana y no sólo realizó las mediciones correspondientes en los criminales, sino que también se preocupó por conocer la historia de los delincuentes, lo que finalmente ayudó a formar sus propios juicios y conclusiones con respecto a los criminales mexicanos.

No se ha determinado en qué momento Roumagnac decidió estudiar la criminalidad en el país, sin embargo, gracias a un escrito autobiográfico se sabe que en 1888, con aproximadamente 18 años, estaba estudiando ingeniería en la Escuela de Minería, cuando por razones que no explica tuvo que ingresar al mundo del periodismo en El Eco del Universal, de Manuel Caballero, "mi primer maestro, en la para mí nueva ciencia". ${ }^{13}$ Antes de ese momento, su acercamiento a la prensa, y sobre todo a la cárcel, había sido nulo, pues pese a saber de la existencia de ambas, como estudiante no tenía tiempo de leer el periódico y "mucho menos dinero para comprarlo, ya que un periódico costaba lo mismo que [un] atole con tamales o un café con huesitos de manteca". ${ }^{14}$

Fue así que un día, al llegar a las oficinas de El Eco, encontró todo vacío, pues habían arrestado al director del periódico, Manuel Caballero, y al redactor responsable, Rafael Aguilar Marroquí. Su arresto había sido por la reproducción de un artículo de un periódico de California donde se difamaba a una persona. A decir de Roumagnac, la verdadera razón había sido la publicación de una crítica a la primera reelección de Porfirio Díaz y a los festejos que hicieron por ella, los motejados como "los farolitos". ${ }^{15}$

\footnotetext{
12 Lombroso, Los criminales, p. 124.

13 Roumagnac, "Mis recuerdos", p. 5.

14 Roumagnac, "Mis recuerdos", p. 5.

15 Prida, iDe la dictadura!, p. 97.
} 
Los responsables de la crítica fueron llevados a Belem, donde Caballero enfermó de tifo, lo cual impactó a Roumagnac, pues, aun cuando su maestro logró recuperarse, El Eco no volvió a publicarse:

Y así fue cómo, como estudiante de Minería que empezaba a aprender del periodismo las lecciones color de rosa: crónicas teatrales con pase libre, entrada a los camerinos de artistas y sonrisas de tiples agasajadas, empecé también a darme cuenta de que la cárcel, de que ese Belem [...] no sólo era algo que servía para segregar criminales, sino también para encerrar periodistas. ${ }^{16}$

Para la última década del siglo XIX, Roumagnac fue acercándose cada vez más a las cárceles y a los criminales, pues en 1892, como reportero del periódico El Tiempo, estuvo presente en el fusilamiento de Bruno Martínez, y en 1896 regresó a Belem, aunque ahora por tres meses en calidad de preso por la publicación de un artículo en El Globo, donde había hecho mofa y "denunciaba la incapacidad y corrupción que existía en el batallón de Zapadores". ${ }^{17} \mathrm{Al}$ salir de la prisión, Roumagnac ingresó a la policía y comenzó sus estudios como criminalista, de lo que se derivó la obra ya mencionada.

Su estudio de Los criminales en México no sólo refleja el pensamiento que una época y un grupo social tuvo sobre los criminales, sino también habla de cómo era la vida en la cárcel de Belem, pues su autor se ocupó de conocer la forma en la que vivían los presos, aunque no con la intención de darlo a conocer al público, sino como parte de su investigación para la cual era importante saber cuáles eran las costumbres de los delincuentes. $\mathrm{Al}$ inicio de su trabajo Roumagnac señaló su adhesión a la escuela antropológica criminal y definió que ésta tenía "que ser el examen detenido y la definición exacta, en primer lugar, de los crímenes cometidos por individuos atacados de una enfermedad positiva, como la locura y la epilepsia, y en segundo, de las formas de organización mental defectuosa, que son el resultado de una mala herencia". ${ }^{18}$

Los defectos por herencia no sólo eran cuestión de atavismo, ya que desde Lombroso se consideraba que gran parte de éstos eran "en primer término de la edad avanzada de los padres, del alcoholismo, de la irritabilidad del padre, y en segundo lugar de la alimentación y criminalidad de

\footnotetext{
16 Prida, ¡De la dictadura!, p. 5.

17 Barrón, "Carlos Roumagnac", p. 173.

18 Roumagnac, Los criminales, p. 12.
} 
los progenitores". ${ }^{19}$ Para Roumagnac estas consideraciones, así como la estatura, las medidas del busto, el tronco, el perímetro del pecho, el largo de los dos miembros superiores, el tamaño de la mano, el pie, la huella, la forma y el tamaño del cráneo, la longitud y forma de la cara, nariz, orejas, ojos, cabello, barba, la fisionomía del rostro -si era feo o hermoso-, el parentesco, los hábitos de la familia, la infancia del criminal, sus tendencias, la forma en que había preparado y ejecutado el crimen, su estado de ánimo después de delinquir y finalmente su raza, edad y lugar de nacimiento constituían la ficha signalética que daba pie al estudio antropológico del criminal que plasmó en su libro.

Es importante señalar que Roumagnac no siguió íntegramente a Lombroso, sino que retomó algunos planteamientos del italiano Enrico Ferri. Uno de ellos fue la investigación de los hábitos de la familia y del delincuente, pues el italiano había postulado que las causas del delito no eran exclusivas de la fisionomía del criminal, sino que también podían ser causas los medios físicos y sociales en los que había crecido. ${ }^{20}$ Probablemente la inclusión de estas nuevas propuestas de la antropología criminal que siguió Roumagnac en 1903 se debió a las numerosas críticas que había tenido esta escuela en diferentes partes de Europa por su determinismo, lo que la obligó a aceptar nuevos planteamientos como fue "la influencia de factores ajenos al organismo del criminal". ${ }^{21}$

Para el criminalista mexicano, el delincuente era un enfermo que en ciertos casos debía aislarse y en otros tantos podía curarse. Todo dependía del tipo de transgresor de que se tratara, pues en su opinión existían tres tipos de criminales: el primero era el "criminal por influencia de raza", ${ }^{22}$ que había cometido algún delito porque era un criminal nato, fuera por atavismo, herencia directa o por alguna enfermedad congénita. Este tipo de persona debía ser aislado o sometido a una terapia rigurosa pues no podía curarse. El segundo era el criminal por influencia de medios, quien delinquía por no haber tenido educación, aunado al hecho de que había vivido en un medio vicioso o se había "contagi[ado] de otros seres dañados"; ${ }^{23}$ el tercero era el criminal por influencia del momento, es decir, el que había delinquido al dejarse dominar por la pasión. Para Roumagnac, éste y el segundo tenían alguna posibilidad de regeneración, porque el medio los había influido y debían aplicárseles "los medios

\footnotetext{
19 Lombroso, Los criminales, p. 17.

20 Speckman, Crimen, p. 95.

21 Speckman, Crimen, p. 95.

22 Roumagnac, Los criminales, p. 59.

23 Roumagnac, Los criminales, p. 59.
} 
a la vez que represivos, preventivos" ${ }^{24} \mathrm{y}$, sobre todo, se les tenía que alejar del pulque y educar antes de que llegaran al crimen, pues era un "remedio sin duda más eficaz que la pena de muerte [y] que las cárceles tal como están ahora."

Además de estas ideas, otros autores pensaron que algunos signos de la criminalidad se podían ver en la fealdad: "el rostro de este tipo es grosero, anguloso, estúpido: el color sucio. Las mujeres son feas de formas, de facies y movimientos; todos tienen una expresión tan siniestra como repulsiva"; ${ }^{25}$ los tatuajes, la desviación sexual -debido a que ambos eran un signo más de su conducta anormal-, y el lenguaje. ${ }^{26}$ Según señalaban, los criminales usaban un lenguaje particular entre sí para no ser entendidos por los otros, idea proveniente de los estudios de Lombroso. ${ }^{27}$

Los criminólogos positivistas pusieron en práctica las ideas de dicha escuela mediante los gabinetes antropométricos en las cárceles. De ello resultó la confirmación de la idea de que los criminales natos no tenían cabida en el organismo social, pues en ellos jamás podrían ejercitarse los órganos que detenían los impulsos malos del hombre y que en los demás eran susceptibles de generar la estimulación de los sentimientos altruistas y, por ende, un buen desarrollo de la sociedad. ${ }^{28}$

En algunos lugares de Europa esta escuela no tuvo tanto éxito, pues desde el primer congreso de antropología criminal en 1886, la escuela francesa de sociología criminal se presentó como su principal contraparte. La escuela francesa no atribuía el delito a las características físicas del delincuente, sino a factores ambientales, sociales y culturales. ${ }^{29} \mathrm{En}$ México, su único representante fue Julio Guerrero, quien en 1903 publicó La génesis del crimen en México, en la que explicaba que el ser humano libraba una lucha constante contra los agentes naturales y sociales que lo rodeaban. Muchos fracasaban en el combate, pues sus características no los hacían seres aptos para luchar de la misma forma en que lo hacía la sociedad en su conjunto. El resultado era que estos individuos se convertían en obstáculos inevitables que producían "el fenómeno de la lucha abierta por la existencia, en la forma genuina de un episodio de la evolución animal." 30

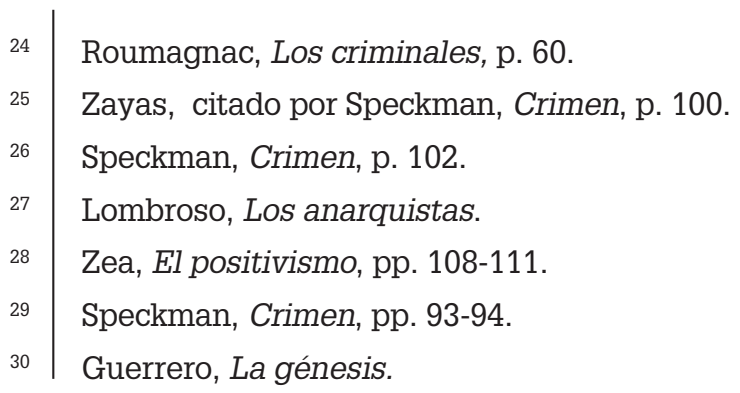


Estas ideas tuvieron algunos detractores en México, como Antonio Verdugo, quien pertenecía a la escuela liberal clásica. Verdugo argumentaba que todos los hombres eran iguales entre sí, aunque tenían necesidades y tendencias diferentes, pero ayudaban a constituir una sociedad universal en la que todos debían asistirse por el respeto al derecho, pues éste era una expresión de los derechos y las obligaciones de los diferentes individuos que existían para mantener la armonía y la alianza entre todos. Cuando esta alianza era rota por alguno de los individuos, la legislación penal se encargaba de "restablecer el equilibrio perturbado por el delito, mediante la imposición de un castigo, en nombre de la ley". ${ }^{31}$

Para Verdugo, el delito no era un acto cometido por un determinado grupo de personas, sino que cualquiera de los individuos que constituía a la sociedad podía realizarlo, pues Dios había creado en todos los humanos una conciencia que permitía entender la diferencia entre los actos justos e injustos, para que así cada uno fuera responsable de sus acciones. Esta creencia lo llevó a criticar por un lado a Spencer y al organismo social, pues según él dicho autor explicaba el derecho mediante la ciencia y diversos aspectos materiales, sin tener en cuenta que existía algo superior al hombre; y por el otro a Lombroso y Ferri, porque, uno con los estigmas físicos y el segundo con los factores sociales, eximían al individuo de su responsabilidad en la sociedad, negando la posibilidad de lo que Verdugo denominaba "criminalidad interna", es decir, si el criminal experimentaba remordimiento, si se había arrepentido o si había sido consciente de su acto.

Ahora bien, Verdugo analizó la obra de los mencionados autores y señaló que los criminalistas sustentaban que sólo los criminales tenían anomalías en el tamaño de los hemisferios, mientras que los "normales" tenían ambos hemisferios regulares y normales, lo que rebatió al señalar que estas anomalías craneales también se habían encontrado en "cerebros de hombres de letras, muy distinguidos y sobre todo inocentes de todo delito." Por éstas y otras características atribuidas a los criminales, retoma la postura del doctor Duisson, que decía:

De creer a ciertos autores, [...] la capacidad craneana sería más grande en el asesino que en el ladrón; el primero sería braquicéfalo y el segundo dolicocéfalo; el asesino tendría la nariz encorvada y el ladrón remangada; el uno presentaría la mirada vaga y fría, mientras que el otro la tendría oblicua ó errante. ¿Cómo explicar entonces un hecho que está fuera de duda, a saber, que la mayor parte de los criminales

${ }_{31}$ Verdugo, La responsabilidad, pp. 6-10. 
comienzan por el robo y acaban por el asesinato? ¿Habrá que admitir que el ladrón cambia de nariz haciéndose asesino? ${ }^{32}$

Finalmente, la mayor crítica de Verdugo va dirigida al concepto de atavismo, pues éste condenaba a la sociedad "a ser siempre la impasible espectadora del crimen, [...] sin ningunos medios por parte de las gentes honradas para poner los diques, o por lo menos derivarlo en sus inevitables devastaciones." 33 Argumenta que si el concepto fuera cierto, la escuela, las nociones del honor y del deber, el buen comportamiento, el esfuerzo individual y sobre todo la formación de una familia serían acciones superfluas, "una risible fantasía [donde ninguna] influencia [podría] evitar esas fatalidades hereditarias que [...] persisten a través de las generaciones de una manera necesaria, produciendo aquí la locura, allá el suicidio, acullá la mendicidad". ${ }^{34}$

La prensa mexicana siguió de cerca estas discusiones y los trabajos de Lombroso con el fin de encontrar una explicación sobre el origen de la criminalidad. Algunos artículos, como los del periódico El Universal posteriores a 1893, señalaban que si bien las teorías lombrosianas no eran aún comprobadas en su totalidad, tenían por lo menos de cierto el hecho de que al criminal nato lo caracterizaba una facie especial, lo cual se debía a que

los hábitos de nuestro espíritu se reflejan en nuestra fisionomía; de igual manera ciertas disposiciones físicas, especiales, corresponden a inclinaciones naturales, particulares; todo lo cual nos permite formarnos una opinión de un individuo por sólo su rostro que refleja su personalidad y sus pasiones dominantes. ${ }^{35}$

No obstante, el mismo periódico, antes de la llegada de Ramón Prida a su dirección, había calificado las ideas del criminalista italiano como erróneas, pues si bien éste reconocía en el cráneo pequeño signos de los asesinos, lo cierto era que "desgraciadamente estos signos faltan en muchos criminales y se encuentran entre muchas gentes de bien. [Ejemplo de ello eran] el cráneo de Lammennais y el de Gambetta [pues] eran muy pequeños y ni Gambetta ni Lammenais fueron asesinos". ${ }^{36}$ Además, se

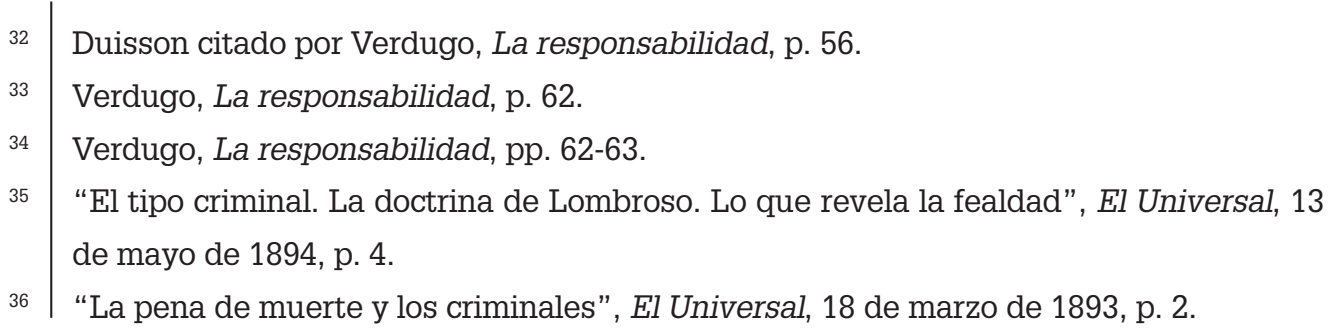


señala que era imposible que las teorías de Lombroso se comprobaran porque todos los criminales eran distintos entre sí, pues cada uno había cometido una infracción diferente ante una ley o una costumbre especial, lo que generaba que las diversidades del crimen fueran infinitas, pues el ser humano es criminal por naturaleza, ya que mata desde la prehistoria, y sólo había dejado de hacerlo gracias al esfuerzo que había traído consigo la lenta edificación de la moral. ${ }^{37}$

En 1892, en otro artículo del mismo diario se señalaba que se hacía un uso indiscriminado de las escuelas criminalísticas y que si bien antes los jurados estaban compuestos por personas a las que se les pedía criterio y conciencia, en ese momento se requerían personas que conocieran a Lombroso, Garófalo y Maudsley y que sobresalieran en química, biología, higiene, patología, historia natural, matemáticas, psicología, sociología, economía política, entre otras especialidades pues "solamente así podrá llegar a decidir si un individuo que ha asesinado, incendiado, robado, estuprado, violado y atacado es culpable de acuerdo con estos importantes ramos del saber humano". 38

El autor continuaba su crítica porque veía en estas escuelas a las culpables de que los defensores y los jueces se volvieran coleccionistas de datos y clasificaciones con los que planeaban formarse un criterio para impartir justicia. La situación había llegado al extremo de que en un sinfín de ocasiones se presentaba al criminal hasta en "paños menores," pues a partir de sus características físicas se explicaban cuáles eran sus enfermedades y sobre todo su genealogía, de la que

resulta que el bisabuelo del delincuente padeció en sus primeros años una fuerte diarrea, a consecuencia de un golpe que le profirió su nodriza; [ergo,] está demostrado que debía matar a cualquiera de sus conciudadanos, con una necesidad fatalmente "expansible" del crimen. ${ }^{39}$

De todo ello resultaba que atribuían diferentes características y teorías sobre el crimen y que conforme al último libro leído, era "el placer de fabricar un criminal", en el que se perdía el verdadero punto de la justicia debido a que las causas físicas que habían determinado al criminal parecían demostrar y justificar su inocencia.

\footnotetext{
37 "La pena de muerte y los criminales", El Universal, 18 de marzo de 1893, p. 2.

38 "La criminalidad y las nuevas teorías. Razas de delincuentes. Responsabilidad de nuestros padres", El Universal, 3 de agosto de 1892, p. 2.

39 "La criminalidad y las nuevas teorías. Razas de delincuentes. Responsabilidad de nuestros padres", El Universal, 3 de agosto de 1892, p. 2.
} 
Empero, esta "fabricación de criminales" no era privativa de las escuelas criminalistas, pues a decir de Robert Buffington durante el porfiriato se buscó al "tipo" clásico del criminal con la finalidad de poder proteger a la sociedad moderna ${ }^{40}$ y cada grupo o cada persona tenía alguna idea sobre la razón por la cual existían los criminales. Ejemplo de ello fue en primer lugar el caso del licenciado Agustín Borges, agente del ministerio público en 1892 y procurador de justicia en 1896, quien explicó que el índice de criminalidad había aumentado de 1891 a 1892 debido a la disminución de los matrimonios; ${ }^{41}$ en segundo, la postura de El Universal de Ramón Prida, que denunciaba que si la delincuencia había aumentado hacia 1895 esto era porque los filántropos, en vez de ayudar a la sociedad, eran "llorones insulsos y compasivos ciegos y torpes" que acostumbraban fomentar la pereza, la mendicidad, así como las malos hábitos, pues por algunos de ellos los delincuentes no se castigaban de la forma en la que se debía, lo que originaba que otros siguieran su ejemplo. ${ }^{42}$

En la perspectiva del mismo diario, esta situación se agudizó en 1896 porque la policía y la administración de justicia habían sido contagiadas por los filántropos, de lo que había derivado que los castigos disminuyeran o que simplemente no se cumplieran, y con ello los hombres proclives a la delincuencia habían aumentado, pues ya no tenían motivos para sentir temor al castigo. ${ }^{43}$

Por su parte, El Demócrata asociaba el crimen con la temperatura, pues con ayuda de los policías se descubrió que a mayor frío, menos crímenes; con lluvia, ninguno; mientras que con más calor aumentaban. ${ }^{44}$ Finalmente, otros autores consideraban la falta de instrucción y el alcoholismo como los factores determinantes para el desarrollo de la criminalidad, pues en un pueblo sin instrucción no podía haber orden, mientras que los padres alcohólicos sólo podrían engendrar hijos alcohólicos, ya que la ebriedad era considerada un problema de herencia que determinaba "la propensión a la delincuencia". ${ }^{45}$

En tiempos recientes se ha señalado que la mayor preocupación por los crímenes y sus actores se dio hacia 1900, cuando en la ciudad de Mé-

\footnotetext{
40 Buffington, Criminales, p. 58.

41 "El crimen y el jacobinismo", El Universal, 28 de marzo de 1893, p. 2.

42 "La criminalidad y la ley", El Universal, 23 de octubre de 1895, p. 1.

43 "La criminalidad en México. Más justicia y más policía", El Universal, 18 de abril de 1896, p. 1.

44 "Criminalidad termométrica", El Demócrata, 10 de enero de 1895.

45 Speckman, Crimen, p. 87.
} 
xico se percibió un aumento importante en la criminalidad, y que fue en las primeras décadas del siglo xx cuando "criminólogos, la policía y el Poder Judicial emprendieron la identificación de delincuentes y los aislaron de los ciudadanos "decentes". Al hacerlo, convirtieron en sospechosos a todos aquellos que parecían alejarse de las costumbres modernas". ${ }^{46}$

Sin embargo, en 1892 aún faltaba definir cómo y quién era físicamente el delincuente nacional. Para llegar a identificar a dicho sujeto habría que medirlo, conocerlo y fijar sus peculiaridades. Con este objetivo se establecieron dos gabinetes antropométricos el mismo año, el primero en la cárcel de Belem y el segundo en la penitenciaría de Puebla, bajo la dirección de los médicos Francisco Martínez de Baca y Manuel Vergara.

\section{El sistema Bertillon y el gabinete antropométrico de Fernández Ortigosa}

La propuesta de establecer el primer gabinete antropométrico en México surgió del médico legista Ignacio Fernández Ortigosa, quien publicó en 1892 su Memoria sobre la identificación científica de los reos. Dedicó el trabajo al Presidente Porfirio Díaz, con la finalidad de que aceptara que el autor introdujera el sistema Bertillon en la cárcel de Belem, ya que ese sistema era usado en Francia y otros países de Europa "con maravillosos resultados", ${ }^{47}$ por lo que también debía de traerse a las cárceles mexicanas. ${ }^{48}$

Para sustentar sus propuestas, Ortigosa publicó en sus Memorias diversas cartas de personalidades importantes en el ámbito jurídico como Agustín Borges, Procurador de Justicia; Rafael Rebollar, magistrado de la sala de casación del Tribunal Superior de Justicia del Distrito Federal; Manuel F. de la Hoz, juez $2^{\circ}$ de lo criminal; y Agustín Verdugo, oficial de Instrucción del Instituto de Francia.

Para Agustín Borges, quien había visto en la disminución de los matrimonios el aumento en la criminalidad, era de suma importancia introducir en México las ideas de Alphonse Bertillon, doctor y criminalista parisino, creador del sistema del mismo nombre, porque sólo así podría aplicarse el castigo correcto. Borges consideraba que la forma de identificación que se tenía en aquel momento era ineficaz por la forma en la que se castigaba

\footnotetext{
46 Piccato, Ciudad, p. 21.

47 Fernández, Memoria, p. 3.

48 Cristina Rivera Garza considera que dicho sistema "reveló la penetración de la influencia europea en el desarrollo de formas locales de supervisión". Rivera, La Castañeda, p. 197.
} 
a los reincidentes, ya que ignoraba quiénes eran y los dejaba pasar como delincuentes primerizos, cuando en la mayoría de los casos los criminales eran reincidentes comunes, pues bastaban dos o más actos para que en ellos se arraigara el hábito de delinquir. De implementarse estas ideas en las cárceles del país, se podría establecer quiénes eran los delincuentes y, sobre todo, los reincidentes, a los que finalmente se podría aplicar el castigo merecido, pues la reincidencia se considera un agravante en el momento de dictar la sentencia. ${ }^{49}$ Entre tanto, lo que Bertillon proponía era un sistema, es decir, un conjunto de reglas o principios "racionalmente enlazados entre sí", ${ }^{50}$ con lo que se aseguraba que la ciencia ayudaría a castigar a los delincuentes y sobre todo a encontrar a los reincidentes, pues se pensaba que la propuesta de Bertillon, al ser un sistema comprobado, no tendría fallas y que fácilmente se podría encontrar al "tipo de criminal mexicano."

Estas ideas eran similares a las de Rafael Rebollar, quien además agregaba que los medios utilizados entonces la mayoría de las veces sólo daban como resultado mentiras, mientras que el sistema nuevo sería infalible pues "tiene a la ciencia como colaboradora; [...] es resultado de la observación y de grupos de hechos y grupos de fenómenos que, sometidos a métodos, pagan tributo al fin propuesto". ${ }^{51}$

Manuel de la Hoz confirma la idea de Rebollar. Para él, el sistema que se pretendía establecer era un método científico indiscutible, lo que ayudaría a que el aparato de justicia funcionara de manera correcta; la identificación que se realizaba en esos momentos resultaba confusa, lo que derivaba en graves errores, pues en ocasiones el delincuente primerizo era confundido con el reincidente. Estas situaciones eran los resultados del mal sistema de identificación que se tenía, dado que la mayoría de los criminales provenían de las clases bajas, donde la mayoría de los individuos eran indígenas

que tienen los signos característicos de un tipo siempre uniforme y muy poco variado, [de lo que] sucede que el que pretenda identificar a un individuo de la raza indígena en un cuartel, por ejemplo, en donde el soldado tiene la misma talla e igual uniforme, no podrá conocer al hombre que busca. ${ }^{52}$

\footnotetext{
49 Carta de Agustín Borges, Fernández, Memorias, pp. 5-6. http://lema.rae.es/drae/?val=sistema, consultado el 5 de febrero de 2015. Carta de Rafael Rebollar, Fernández, Memoria, p. 8.

52 Carta de Manuel de la Hoz, Fernández, Memoria, p. 11.
} 
Por su parte, Agustín Verdugo, el abogado liberal que estaba en contra del determinismo de las escuelas criminalísticas, en su colaboración cuida mucho sus palabras, pues el sistema Bertillon se asociaba continuamente con ellas, por lo que expone que si bien existían teorías que buscaban averiguar la responsabilidad de un delincuente, éstas no eran sino conjeturas de las que no podía obtenerse un veredicto, mientras que el nuevo sistema permitiría identificar a cada delincuente, lo que ayudaría a los jueces a fijar una sentencia porque sabrían con seguridad si el procesado era reincidente o no. ${ }^{53}$

Finalmente, Ortigosa agregó a su Memoria la propuesta que había formulado el abogado Adolfo Díaz al ayuntamiento de la ciudad de México el 10 de marzo de 1892, en la que argüía que el sistema de identificación de reos ya se usaba en diferentes partes del mundo con mucho éxito, y principalmente en la Prefectura de París, donde se había originado y en el que Ortigosa había adquirido y perfeccionado sus conocimientos con el mismo Bertillon. Además, explicaba que esta implementación traería un gran beneficio al sistema de justicia y por ende a la sociedad, pues en

la Cárcel de Belem, si no estoy mal informado, se practica la identificación de los presos valiéndose de sus generales y de media filiación; a reserva de que en el Juzgado respectivo se complete con las fotografías que de los reos se sacan, las generales pocas veces dan resultado, pues queda al arbitrio del interrogado contestar o no la verdad y siempre la oculta o la desfigura intencionadamente, puesto que la mayor parte de las veces está interesado en desviar la acción de la Justicia. ${ }^{54}$

A los motivos explícitos se añadió otro factor a favor: el hecho de que el sistema Bertillon fuera de origen francés, pues durante la época que aquí se trata todo lo de origen francés representaba el adelanto social y la modernización de los sistemas; para el caso de la medicina,

la influencia francesa se dejó ver en toda su magnificencia en las clases urbanas, que vestían a la moda francesa, leían francés porque era considerado el idioma de la cultura, viajaban a Francia a preparase en diversas profesiones. En materia de salud, el Estado adoptó los códigos y disposiciones franceses de salubridad. ${ }^{55}$

\footnotetext{
53 Carta de Agustín Verdugo, Fernández, Memoria, p. 12.

54 Carta de Adolfo Díaz, Fernández, Memoria, p. 14.

55 López, El dolor, p. 39.
} 
Ya en la praxis, la mayoría de los motivos para cambiar el sistema de identificación de reos que había estado vigente desde el 11 de enero de $1842^{56}$ se sustentó en el hecho de que el que operaba en ese momento no respondía a un método científico, pues se basaba en observaciones subjetivas, de las que en la mayoría de las ocasiones se obtenían resultados falaces. La identificación se obtenía mediante la anotación de los datos biográficos generales, la media filiación y dos fotos del preso, de las que ya en la realidad ninguna podía usarse pues éstos procuraban hacer muecas frente a la cámara con la finalidad de imposibilitar una buena toma. Sobre los registros se escribía el nombre que los mismos presos habían proporcionado y que, como señala entre otros Manuel de la Hoz, en innumerables ocasiones resultaba falso, pues ocultaban sus datos verdaderos para no ser identificados como reincidentes.

Los datos generales se obtenían a través de las siguientes preguntas: ¿Cómo se llama usted?, ¿de dónde es?, ¿es usted casado, soltero o viudo?, ¿cuántos años tiene?, ¿cuál es su ocupación?; finalmente, ¿dónde vivía antes de ser aprehendido? De lo "que queda al arbitrio del interrogado contestar á estas preguntas de acuerdo con la verdad o de acuerdo con su capricho", ${ }^{57}$ mientras que la media filiación quedaba a criterio de algún encargado de la alcaldía y de algún otro del juzgado, pues a cada preso se le realizaban dos medias filiaciones.

La primera de estas medias filiaciones se efectuaba en el juzgado después de que se dictaba sentencia al acusado, mientras que la segunda quedaba a cargo de algún escribiente de la cárcel en el momento en que el sentenciado ingresaba como preso, y resultaba que no fuese raro que ambas medias filiaciones de un mismo preso no se parecieran entre sí. ${ }^{58}$ En ellas los empleados debían calificar si el individuo era alto, bajo o regular; si tenía pelo y cejas negros, castaños, canos o entrecanos; si su frente era regular, grande o chica; si sus ojos eran claros, negros o pardos; su nariz regular, chata o aguileña; si su boca era grande, regular o chica; su barba negra, castaña, cana o entrecana, escasa o poblada, y finalmente se debían anotar las "particulares" del preso, es decir, todas las marcas que ellos alcanzaban a distinguir, fueran lunares, cicatrices, etcétera.

Respecto de este sistema, el propio Lombroso había expuesto en su obra la forma en que ayudaba a ubicar a los criminales, ya que en algunas ocasiones había gente inocente que era tomada por criminal, lo que sólo

\begin{tabular}{l|l}
${ }_{56}$ & Fernández, Memoria, p. 19. \\
${ }_{57}$ & Fernández, Memoria, p. 14. \\
${ }_{58}$ & Fernández, Memoria, p. 18.
\end{tabular} 
se podría dilucidar mediante un estudio antropométrico. Para ejemplificar este punto relató el caso de un hombre de apellido Rossi que había sido condenado a cadena perpetua al habérsele confundido con un salteador de caminos. Sin embargo, el hombre era una persona honrada, lo que se descubrió mediante el examen antropométrico; gracias a esto Rossi recuperó la libertad. ${ }^{59}$

Así, los males del sistema de identificación mexicano se pensaban remediar mediante el sistema Bertillon, pues se creía que no tendría fallas porque la identificación resultaba de la medición de diversas longitudes "huesosas" del criminal, que ya no crecen a partir de los 20 años. Ortigosa señalaba que para poner en marcha el sistema se necesitaba un compás fabricado por M. Colás, tres estantes para las fichas de identificación de los hombres y tres para las mujeres, varios cartabones de tamaños grande, mediano y pequeño, un compás de cremallera especial, un banquillo y una jaladera fijada en un mueble con la finalidad de que los individuos pudieran sostenerse mientras se les realizaban algunas mediciones. ${ }^{60}$ Además eran necesarias dos habitaciones, una para la sección de antropometría y otra para la de fotografía.

En la sección de antropometría se debían tomar, anotar y clasificar las medidas de diversas longitudes huesosas, que eran el diámetro de la cabeza, el diámetro transverso de la cabeza, la longitudinal del dedo medio izquierdo, la del pie izquierdo, la del codo, la estatura (longitud), la anchura de la oreja, la estatura y la braza. Con las cifras obtenidas se debía hacer una clasificación de éstas según fueran grandes, medianas o pequeñas, las cuales correspondían a cada uno de los estantes. Estos estantes debían estar divididos por cajones donde se anotaban los promedios de las mismas cifras que contenían al frente de ellos, con la finalidad de que aquel que buscara o deseara clasificar a un acusado pudiera hacerlo mediante un sistema de eliminación de medidas, y no por nombre como se hacía antiguamente.

Se calculó que cada cajoncito albergaría un promedio de 500 cartones, los que a su vez estarían divididos por cartones de colores que marcarían las divisiones de medidas grandes, medianas y pequeñas de la longitud de oreja, de anchura de oreja, de talla y, finalmente, de la braza, aunque para "hacer más fácil el trabajo de eliminación, las divisiones en cada cajilla se harán siempre de manera que las grandes longitudes estén colocadas del lado del fondo de la casilla". ${ }^{61}$ Cada cartón estaría dedicado

\footnotetext{
59 Lombroso, Los criminales, p. 124.

60 Fernández, Memoria, pp. 20- 28.

61 Fernández, Memoria, p. 27.
} 
a un individuo y en él se anotarían sus longitudes, se agregaría un par de fotografías -de frente y de perfil-, sus generales y el nombre de sus padres, una descripción de su fisionomía y, finalmente, un espacio dividido en seis partes donde se indicarían sus señas particulares según fuera la región donde se encontraran, que debía ser anotado mediante abreviaturas.

V.gr. Cicatriz de 8 milímetros de longitud, en la mitad izquierda de la frente, oblicua hacia abajo y á la derecha, regular o irregular, consecutiva a herida hecha con instrumento cortante o contundente y deforme. Un lunar pigmentado y cubierto de vello en el lado izquierdo de la barba de 3 centímetros de longitud por 12 milímetros de anchura.

Todo lo cual quedará reducido por medio de abreviaturas a lo siguiente: C.0, S.f.izq.ob.ab.d.r. ó ir. cont. ó cort. def-l. pl br izq. 3 x $12 .{ }^{62}$

En el departamento de fotografía se podría recurrir al fotógrafo con quien la Junta de Vigilancia tenía contrato, con el objetivo de que esta sección no generara mayores gastos. Únicamente éste debía aprender y ajustarse a las técnicas del retrato judicial que se usaban internacionalmente, en que se consideraba la unidad de la luz, de reducción, de tiempo de exposición, de posición del sujeto y de forma y tamaño, pues estas características daban uniformidad a los diferentes retratos, al tiempo que mostraban los ángulos que se necesitaba identificar. El fotógrafo sería ayudado por dos personas, las cuales formarían parte del equipo de ocho que se necesitaba para instalar dos departamentos del gabinete antropométrico en Belem. El personal administrativo quedaría de la siguiente forma: un jefe de servicio, un encargado de medidas, dos escribientes, tres fotógrafos y por último un empleado encargado de la clasificación alfabética.

La Memoria llegó a manos del presidente Porfirio Díaz, quien aprobó la solicitud del médico Ortigosa, como también lo hizo el ayuntamiento de la ciudad de México el 17 de octubre de ese año. Por conducto de la Secretaría de Justicia e Instrucción Pública, el presidente señaló que era un deber y una preocupación constante combatir la reincidencia, pues consideraba que en un lugar donde impera el orden público, el reincidente era una figura extraña que era necesario estudiar y combatir. Para que las órdenes del presidente se concretaran, por el mismo conducto se ordenó que se reformara la fracción $3^{\underline{a}}$ del artículo 93 del reglamento de la cárcel del 26 de octubre de 1880, en los siguientes términos:

62 $\quad$ Fernández, Memoria, p. 32. 
Mandar que además de las generales de los reos que con arreglo a la ley deben aparecer en las partidas y procesos, se asegure la identidad de los reos condenados a la pena de prisión por medio de retratos fotográficos, complementados en cuanto fuere posible con las indicaciones del Señalamiento Antropométrico. ${ }^{63}$

El gasto que se debía realizar para la instalación del gabinete antropométrico en la cárcel de Belem fue calculado por el ayuntamiento de la ciudad de México en 8814.15 pesos, los que se pagarían con los fondos de la junta de vigilancia de cárceles y con dinero que se tenía destinado a las habitaciones del alcaide. El presidente de la junta de vigilancia decidió economizar inicialmente 1669.73 pesos gracias a que todos los muebles y útiles de madera que debían emplearse en el gabinete fueron construidos por los presos, con los materiales del taller de carpintería. Al mismo tiempo se ahorraron miles de pesos más, lo que dio como resultado que "con la suma de \$208 he fundado un gabinete antropométrico que llena las condiciones exigidas por el nuevo Código de Procedimientos Penales y por cuyo gabinete alguien te pidió \$8 000" ${ }^{64}$

Además, por órdenes de Salinas y Carbó también se tuvo que ahorrar en el sueldo del jefe del gabinete antropométrico, por lo que a finales de 1894 cuando se terminó la instalación del gabinete no se llamó para este cargo a Ignacio Fernández Ortigosa, sino a Ignacio Ocampo, el médico de la cárcel, quien desde el principio había sido comisionado por Salinas

para que diese principio a los trabajos antropométricos al Sr. Director de la Sección Médica Dr. D. Ygnacio Ocampo, quien con una autoridad y celo dignos de elogio, ha practicado ya la medición de quinientos sesenta y dos sentenciados y continúa midiendo a los demás a razón de diez y seis en cada día. ${ }^{65}$

Esta decisión del presidente de la junta de vigilancia debió tomar por sorpresa a muchos, pues durante 1892, y aún en los primeros meses de 1893, el propio Ortigosa, algunos conocidos suyos y algunos otros interesados en la materia escribieron numerosos artículos en diversos diarios de la ciudad en los que se reprodujo la información de las Memorias, así

\footnotetext{
63 AHDF, 1019.

64 Informe de Salinas y Carbó ante el H. Ayuntamiento, "El gabinete antropométrico de Belem. Las economías del Dr. Salinas y Carbó. Lo que es el método Bertillon", El Demócrata, 21 de mayo de 1895, p. 2.

65 AHDF, 1047.
} 
como la propuesta que se había hecho al ayuntamiento de la ciudad de México y que ponía a Ortigosa como el único hombre capaz de llevar a cabo dicha tarea.

Tal fue el caso del doctor Manuel Flores, quien sostuvo que como los criminales cada día eran más y sobre todo más experimentados en su materia, se necesitaban nuevos y más eficientes medios para controlarlos. Por ello recomendó el uso del procedimiento antropométrico del doctor Bertillon, que servía para identificar a un determinado tipo de delincuente, específicamente al delincuente habitual; a aquel que era propenso e inclinado al crimen por su conducta "invariablemente delictuosa", y que por su naturaleza debía de identificarse, pues, aunque hubiera sido castigado alguna vez, tarde o temprano volvería al crimen y por ende a la prisión. En la cárcel debía de funcionar el sistema mencionado, que había sido traído a México por el médico legista Fernández Ortigosa, "quien lo conoce y lo practica con competencia por haberlo estudiado y ejercitado en París al lado del mismo inventor"; sólo así podría prevenirse un nuevo crimen, gracias a que se brindaría un conocimiento superior a la policía, lo que garantizaría formas más eficientes de represión y de seguridad pública. ${ }^{66}$

Incluso el 9 de junio de 1893, cuando comenzó el periodo de gobierno del general Pedro Rincón Gallardo en el Distrito Federal, se le recordó que la propuesta de Ortigosa debía aplicarse cuanto antes, pues en Estados Unidos ya se estaba practicando el sistema Bertillon desde septiembre 1892, a pesar de que el médico mexicano había presentado antes su propuesta y de que el mismo Ortigosa ya llevaba construidos por su cuenta tres estantes con clasificaciones antropométricas que serían un gasto menos para el ayuntamiento de la ciudad de México. ${ }^{67}$

Sin embargo, como se ha señalado, Ortigosa no fue llamado para trabajar en el gabinete, hecho que no pasó por alto la prensa. En mayo de 1895 se le recordó a Salinas y Carbó que la idea de adoptar el sistema Bertillon no había sido suya, sino del doctor Fernández Ortigosa, quien había estudiado las modificaciones que debía tener este sistema para el caso mexicano desde 1877. Fernández había viajado a París para trabajar y aprender del propio Bertillon y a su regreso había medido él mismo a 800 criminales, a los que además mandó fotografiar para llevar sus registros de forma correcta, sin que por esto hubiera cobrado. Al mismo tiempo, se criticó el gabinete antropométrico establecido debido a que los ahorros de Salinas y Carbó habían resultado en lo siguiente:

\begin{tabular}{l|l}
66 & Lucha a muerte. El arte del bandido y la ciencia. Criminales fisiológicos y criminales de \\
& ocasión", El Universal, 22 de septiembre de 1892, p. 2
\end{tabular} 
Los aparatos de ese gabinete no sirven para el objeto: se toma la estatura en uno de nuestros antiquísimos cartabones de madera, donde una corredera marca la altura con diferencias hasta de 7 u 8 milímetros en cualquier movimiento del procesado; para tomar la longitud del antebrazo o del pie, partes que tienen pronunciadas curvas que pueden variar con la robustez del individuo, no hay aparatos a propósito; el libro de medidas no está en perfecta relación con el álbum fotográfico y en las tarjetas hay defectos capitales. ${ }^{68}$

La prensa no fue la única en mencionar las fallas de la antropometría en Belem, ya que personajes como Juan Comas señalaron que el gabinete nunca llegó a funcionar correctamente debido "a que en lugar de quedar en manos de Fernández Ortigosa fue dirigido por el doctor Ignacio Ocampo, que no estaba familiarizado con la aplicación de técnicas francesas" ${ }^{69}$

En este punto cabe señalar que en 1892, cuando Fernández envió su propuesta, México parecía adelantarse al coro de naciones del continente americano, pues para ese entonces sólo en Buenos Aires existía un gabinete antropométrico (1889), mientras que en Estados Unidos apenas se empezaba a poner en práctica el sistema Bertillon (1892). En el lapso que transcurrió hasta 1895, en Perú (1892), Brasil (1893) y Río de la Plata (1894) se instalaron gabinetes de antropometría que propiciaron diversos estudios sobre los criminales, entre los cuales están los trabajos de Juan Vucetich que pondrían a Argentina a la cabecera de los métodos identificación con la creación del primer gabinete de dactiloscopia. ${ }^{70}$ No obstante, aún queda por analizar la forma en que estos gabinetes fueron usados en sus respectivos países, pues "en ocasiones, los gabinetes sirvieron primero al estudio criminológico que a la identificación" ${ }^{71}$

\section{Conclusiones}

La aplicación del sistema Bertillon cayó en hondas contradicciones con lo estipulado por el Código Penal de 1871, pues mientras éste señalaba que el preso podría llegar a regenerarse mediante el trabajo y la educación, la implementación del primero estaba negando al criminal la posibilidad de enmendarse, pues las características físicas del criminal

\footnotetext{
68 “El gabinete antropométrico de Belem. Las economías del Dr. Salinas y Carbó. Lo que es el método Bertillon", El Demócrata, 21 de mayo de 1895, p. 2.

69 Urías, Indígena, p. 175.

70 Del Olmo, América Latina, p. 144.

71 Speckman, "La identificación", p. 119.
} 
lo conducirían a delinquir irremediablemente al momento de salir de la cárcel. Dichas ideas imposibilitaron que la mirada fuera puesta en la rehabilitación del reo, el cual, al verse sin herramientas para enfrentarse a la libertad, terminaba por delinquir, lo que irremediablemente lo conducía de vuelta a Belem.

Por otra parte, hay que señalar que la identificación del criminal no sólo ayudó a crear la idea de seguridad en las personas, sino que ésta, principalmente, permitió construir la imagen del "otro," pues era en el hombre de manta, pulque y rostro ancestral en el que los positivistas veían al criminal. ¿Olvidaban ellos acaso que los hombres de levita cometían crímenes de cuello blanco? Aquí valdría preguntarnos, ¿cómo justificarían el crimen de esta clase de hombres? Negar su existencia habría sido negar uno de los agravantes del código, el cual marcaba que los hombres instruidos que hubieran cometido un delito serían castigados de forma más severa, pues se pensaba que estos sujetos no podían justificar su crimen.

Finalmente hay que señalar que el éxito que logró la antropología criminal en México no sólo nos habla del determinismo imperante en el siglo XIX o de la construcción del "otro", sino que también nos hace ver que los principales actores en materia judicial desconfiaban de sus sistemas, sus leyes y sobre todo de sus instituciones, pues sabían que el encierro en las cárceles no bastaba para amedrentar al delincuente. Pese a esto, la construcción y apertura de la penitenciaría de Lecumberri, en septiembre de 1900, parecía una luz en el camino.

\section{Siglas y referencias}

AHDF Archivo Histórico del Distrito Federal, Ayuntamiento del Distrito Federal, fondo Cárceles en general.

\section{Fuentes}

Aguirre, Carlos, "Cárcel y sociedad en América Latina: 1800-1940", Historia social urbana. Espacios y flujos, Quito, Eduardo Kingam Garcés, Facultad Latinoamericana de Ciencias Sociales, 2009, pp. 209-252.

Barrón Cruz, Martín

Una mirada al sistema carcelario mexicano, México, Instituto Nacional de Ciencias Penales, 2002.

Barrón Cruz, Martín

"Carlos Roumagnac: primeros estudios criminológicos en México", Revista Cenipec, núm. 22 (enero-diciembre 2003), pp. 165-196.

Buffington, Robert

Criminales y ciudadanos en el México moderno, México, Siglo XXI, 2000. 
Castañeda López, Gabriela

"La frenología en México durante el siglo XIX", Historia y filosofía de la medicina, México, Anales Médicos, 2009, pp. 240-247.

Código Penal para el Distrito Federal y Territorio de la Baja-California sobre delitos del fuero común y para toda la República sobre delitos contra la Federación, México, Imprenta del Gobierno en Palacio, 1871.

Flores Flores, Graciela

"La configuración del individuo moderno a través de la institución penitenciaria: cárcel de Belem (1863-1900)", Universidad Nacional Autónoma de México-Facultad de Filosofía y Letras, tesis de Licenciatura en Historia, 2006.

Guerrero, Julio

La génesis del crimen en México: estudio de psiquiatría social, prólogo de Arnoldo Kraus, México, Consejo Nacional para la Cultura y las Artes, 1996.

Hale, Charles A.

La transformación del liberalismo en México a fines del siglo XIX, México, Fondo de Cultura Económica, 2002.

Lombroso, César

Los anarquistas, Madrid, Impresores de la Real Casa, 1894.

Malo Camacho, Gustavo

Historia de las cárceles en México, México, Instituto Nacional de Ciencias Penales, 1987.

Olmo, Rosa del

América Latina y su criminología, México, Siglo XXI, 1981.

Padilla Arroyo, Antonio, De Belem a Lecumberri. Pensamiento social y penal en el México decimonónico, México, Archivo General de la Nación, 2001.

Piccato, Pablo, Ciudad de sospechosos: crimen en la ciudad de México, 1900-1931, México, Centro de Investigaciones y Estudios Superiores en Antropología Social, Fondo Nacional para la Cultura y las Artes, 2010.

Prida, Ramón

¡De la dictadura a la anarquía! apuntes para la historia política de México durante los últimos cuarenta y tres años, El Paso Texas, Imprenta de "El paso del norte", 1914.

Rivera Garza, Cristina

La Castañeda. Narrativas dolientes desde el Manicomio General. México, 1910-1930, 2ª ed., México, Tusquets, 2010. 
Roumagnac, Carlos

Los criminales en México: ensayo de psicología criminal seguido de dos casos de hermafroditismo observados por los señores doctores Ricardo Egea [e] Ignacio Ocampo, México, El Fénix, 1904.

Roumagnac, Carlos

"Mis recuerdos de Belem", El Nacional Dominical, 5 de marzo de 1933, p. 5.

Speckman Guerra, Elisa

Crimen y castigo. Legislación penal, interpretaciones de la criminalidad y administración de la justicia. Ciudad de México, 1872-1910, México, El Colegio de México - Centro de Estudios Históricos, Universidad Nacional Autónoma de México, Instituto de Investigaciones Históricas, 2002.

Speckman Guerra, Elisa

"La identificación de criminales y los sistemas ideados por Alphonse Bertillon: discursos y prácticas", Historia y Grafía, 17, (2001), pp. 99-130. Urías Horcasitas, Beatríz

Indígena y criminal. Interpretaciones del derecho y la antropología en México, 1871-1921, México, Universidad Iberoamericana, Consejo Nacional para la Cultura y las Artes, 2000. 